\title{
Plasma fatty acid patterns derived by principal components analysis and their relationship with cardiovascular risk in a male middle-aged population
}

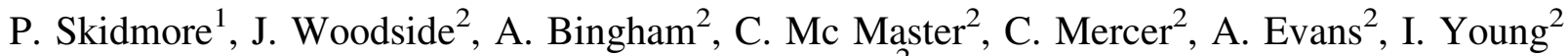 \\ and J. Yarnell ${ }^{2}$ \\ ${ }^{1}$ University of Otago, Dunedin, New Zealand and ${ }^{2}$ Queen's University Belfast, Belfast BT12 6BJ, UK
}

Dietary pattern analysis, usually using data generated from FFQ data, is a valuable tool for investigating relationships between diet and chronic disease ${ }^{(1)}$. Dietary patterns representing diets high in fat have been associated with markers of CVD risk ${ }^{(1)}$. As circulating fatty acid levels also provide an indicator of dietary intake ${ }^{(2)}$ then derived patterns using the same techniques used for creating dietary patterns may also be associated with CVD risk. Circulating fatty acid levels are also related to metabolic disturbances associated with the metabolic syndrome and CVD, as a result of disturbances in the activity of enzymes that catalyse fatty acid desaturation $(\Delta \text { desaturases })^{(3)}$.

Thus, patterns of fatty acids and desaturase activity were determined in plasma and an investigation conducted into how these patterns were related to 10-year CVD risk estimates calculated using the criteria used by the Joint British Societies ${ }^{(4)}$ in a middle-aged male population in the UK. Principal components analysis (PCA) was performed to define fatty acid patterns in 379 men aged 30-49 years. Logistic regression analyses were then carried out to investigate the relationship between these fatty acid patterns and 10-year CVD risk estimates. Three patterns were generated from the PCA: high SFA; high $n$ - 3 ; high MUFA.

Results from logistic regression analyses show that a 1 SD increase in the SFA pattern score was positively associated with an increased likelihood of being in the 10-year CVD risk category (OR 1.71 (95\% CI 1.33, 2.20); $P<0.0001)$ even after adjustment for BMI (Table). There were no significant relationships between scores for the high- $n-3$ and the high-MUFA patterns and category of CVD risk (Table).

Table. OR of having a $\geq 10 \% 10$-year risk of CVD for a 1 sD increase in plasma fatty acid scores

\begin{tabular}{lccccrr}
\hline Model adjusted for & SFA score & $95 \%$ CI & $n-3$ score & $95 \%$ CI & MUFA score & $95 \%$ CI \\
\hline 1. Age & 2.01 & $1.58,2.56$ & 0.88 & $0.72,1.09$ & 0.96 \\
2. Age and BMI & 1.71 & $1.33,2.20$ & 0.84 & $0.67,1.06$ & 0.93 & $0.74,1.18$ \\
\hline
\end{tabular}

These results provide evidence that, as with dietary patterns, the overall combination of fatty acids ingested may be more important in relation to the development of CVD risk, with high levels of SFA associated with higher 10-year risk of CVD.

1. Mente A, de Koning L, Shannon HS et al. (2009) Arch Intern Med 169, 659-669.

2. Ma J, Folsom AR, Shahar E et al. (1995) Am. J. Clin. Nutr 62, 564-571.

3. Vessby B, Gustafsson IB, Tengblad S et al. (2002) Ann N Y Acad Sci 967, 183-195.

4. British Cardiac Society; British Hypertension Society; Diabetes UK; HEART UK; Primary Care Cardiovascular Society; Stroke Association (2005) Heart 91, Suppl. 5, v1-v52. 